\title{
Exploring the sources of 'missingness' in brain tissue monitoring datasets: An observational cohort study
}

\author{
CHARMAINE CHILDS ${ }^{1}$, ANGELA LI CHING NG ${ }^{2}, \mathrm{KUN} \mathrm{LIU}^{3}$, \& JIANXIN PAN 3 \\ ${ }^{1}$ Yong Loo Lin School of Medicine, National University of Singapore, Singapore, ${ }^{2}$ School of Medicine, and ${ }^{3}$ School of \\ Mathematics, University of Manchester, Manchester, UK
}

(Received 10 February 2011; revised 13 fune 2011; accepted 11 fuly 2011)

\begin{abstract}
Objective: To investigate the frequency/category of missing electronic data in export files from patients admitted to the intensive care unit (ICU) with severe traumatic brain injury (TBI).

Research design: Observational cohort study.

Methodology: Patient data-streams for brain temperature $\left(T_{\mathrm{br}}\right)$ and intracranial pressure (ICP) were analysed. Missing data was classified as: missing completely at random (MCAR), missing at random (MAR), missing not at random (MNAR).

Results: Sixty-two patients were studied; $60 \%$ of missing $T_{\mathrm{br}}$ and ICP data events were attributed to electronic data acquisition 'faults'. Missing data rate ranged from 9-43\% (median 25\%). Cross-reference of missing data to clinical observation sheets and medical case notes shows that disconnection of sensors from monitors during critical events are common.

Conclusions and implications for further research: Of concern for clinical management of patients with TBI is the detection of sudden changes in ICP or brain temperature; the parameters which forewarn of impending intracranial catastrophes. Missing data occurred at critical times of the patients stay in the ICU. This work should alert research clinicians of the need to scrutinize monitored physiological data to establish the percentage of missing values in order to obviate bias in the interpretation of results.
\end{abstract}

Keywords: Traumatic brain injury, brain temperature, missing data, categories

\section{Introduction}

Severe traumatic brain injury (TBI) is the leading cause of death and disability in young adults and is forecast to increase worldwide [1]. The medical, surgical and technical resources needed to deliver intensive care to the injured patient during the acute phase after injury, coupled with the costs of longterm neurological rehabilitation, makes recovery from severe brain trauma a significant financial burden for healthcare providers [2].

Continuous monitoring of the injured human brain is key to forecasting and ultimately avoiding catastrophic intra-cerebral events. The introduction of ICP monitoring for avoidance of intra-cranial hypertension is probably the best example of the justification for direct monitoring of the injured brain and has 'spawned' a new generation of measurement technologies to gain an insight to the homeostatic environment of an organ previously inaccessible to the clinician and scientist [3]. Now that the temporal profile of brain oxygen content, brain temperature and regional blood flow can be determined, the scope for diagnosis for impending secondary brain damage is promising [4].

When new sensors are introduced at the bedside for clinical research, the focus is often on the performance of the sensor perse [5], but this is merely a first, albeit necessary, step in a series of events 
(e.g. measurement and monitor-interfacing activities) which ultimately culminate in the production of an end-user physiological database upon which data analysis is undertaken. As it is not a usual practice to assess the performance of each complete bedside system (sensor, sensor-monitor interface cabling, bedside monitor and PC software systems) readings in 'real-time' and displayed on the bedside monitor, will inevitably appear in a different format within a database or spreadsheet. Incomplete data sets, data complications and errors may occur during measurement per se as well as in the electronic and software storage systems which culminate in a measurement reading at a specific point in time. Although spreadsheets are convenient applications for storing and presenting data, checking the datasets for threats to internal validity is the responsibility of the researcher. In previous studies by this group [6] 'empty' data cells were evident in the different data streams within each patient's export file, despite evidence of continuous 'real-time' recordings displayed on the bedside monitor. In some cases a small number of missing data cells were evident, whilst in others large amounts of data appeared to be 'missing'.

Conclusions drawn of the patients' responses to treatment without note of the frequency and type of missing data may lead to measurement bias and erroneous interpretation of the patient's clinical condition. Problems of missing data are well recognized [7]. In clinical trials, methods for handling missing data in very large datasets have suggested that identifying the type of missing data is important to determine the most appropriate way of overcoming bias [8]. In complete case analyses, only patients with full sets of data are included for analysis, but this type of 'case selection' has proven to be a source of bias if incomplete data sets are rejected [7]. On the other hand, in available-case analyses, researchers deal with missing data sets by considering that the patient has a 'zero' value for the particular missing information.

With respect to missing data it is useful to clarify two important terms; the missing data patterns and the missing data mechanisms. Although the terms have specific meanings, researchers sometimes use them interchangeably. A missing data pattern refers to the configuration of the observed and the missing values in a data set, whereas missing data mechanisms describe the possible relationships between measured variables and the probability of missing data. In 1976, Rubin [9] introduced a classification system for missing data problems that is still used in the literature and which refers to three 'missing data mechanisms' to describe how the probability of a missing value relates to the data. Using Rubin's classification, missing data is classified as: missing completely at random (MCAR), missing at random
(MAR) or missing not at random (MNAR) [10]. From a statistical perspective, if the probability of an observation being 'missing' does not depend on observed or unobserved measurements, the observation is Missing Completely At Random (MCAR). If the probability of an observation being missing depends only on observed measurement, the missing observation is Missing At Random (MAR). In the event that observations are neither MCAR nor MAR, they are classified as Missing Not At Random (MNAR), i.e. the probability of an observation being missing depends on unobserved measurements.

Typical examples of MCAR include accidental loss of information and the reason for such 'missingness' events are completely random and unrelated to any patient characteristic. In this case, missing data can be handled by simple techniques without introducing significant bias. An example would be a finding that blood pressure measurements are missing because of a fault with an electronic sphygmomanometer [11]. If data is MAR, subjects with missing values do not show systematic differences from those without missing data but they will have some observed characteristics. For example, missing ICP readings may be lower than the measured readings because those with missing readings may be more likely to have the lower readings. In other words, the reason for the missing values has a 'link' to the specific characteristic of the patient.

Generally, when data is MAR, all simple techniques for handling missing data, including the indicator method and overall mean imputation, give biased results. The third form of missing data occurs when missing values are systematically different from those observed among subjects with other similar characteristics. In this situation, data are not missing at random (MNAR) and no reliable method for correction is available [11]. An example may be sensor failure which may give 'unphysiological' readings.

In view of previous observations of 'gaps' in brain monitoring data sets, the objective of this prospective observational study was to describe the frequency and category of missing neuro-physiological measurements in a cohort of patients with severe TBI (Glasgow coma scale on admission to ICU <8) admitted to the general intensive Care Unit (ICU) of the institution.

\section{Methods}

Approval was granted by the Local Research Ethics committee to conduct the study at the Salford Royal Foundation NHS Trust (Hope Hospital, Salford, UK). In concordance with requirements, the legally 


\begin{tabular}{|l|l|r|r|r|r|r|r|r|r|r|r|r|}
\hline DataDate & DataTime & TMP1 & TMP2 & ICP & CVP & CPP & HR & SaO2 & SAP & DAP & MAP \\
\hline $11 / 03 / 2007$ & $12: 40$ & 38.3 & 37.9 & 25 & 17 & 71 & 86 & 100 & 135 & 77 & 97 \\
\hline $11 / 03 / 2007$ & $12: 50$ & 38.3 & 38 & 23 & 321 & 72 & 88 & 100 & 133 & 77 & 96 \\
\hline $11 / 03 / 2007$ & $13: 00$ & 38.3 & 38 & 25 & 336 & 59 & 86 & 100 & 116 & 64 & 83 \\
\hline $11 / 03 / 2007$ & $13: 10$ & 38.3 & 38 & 24 & 336 & 67 & 86 & 100 & 128 & 71 & 91 \\
\hline $11 / 03 / 2007$ & $13: 20$ & 38.3 & 38 & 24 & 337 & 63 & 87 & 100 & 124 & 71 & 88 \\
\hline $11 / 03 / 2007$ & $13: 30$ & 38.3 & 38 & 27 & 337 & 63 & 87 & 100 & 125 & 73 & 90 \\
\hline $11 / 03 / 2007$ & $13: 40$ & 38.3 & 38 & 25 & 332 & 77 & 84 & 100 & 139 & 84 & 104 \\
\hline $11 / 03 / 2007$ & $13: 50$ & 38.3 & & 26 & 333 & 74 & 84 & 100 & 133 & 79 & 100 \\
\hline $11 / 03 / 2007$ & $14: 00$ \\
\hline $11 / 03 / 2007$ & $14: 10$ & $b$ & $a$ & & & & & & & & & \\
\hline $11 / 03 / 2007$ & $15: 30$ & 38 & & 29 & 273 & 28 & 90 & 100 & 73 & 45 & 57 \\
\hline $11 / 03 / 2007$ & $15: 40$ & 37.9 & 37.5 & 25 & 7 & 93 & 81 & 100 & 151 & 98 & 118 \\
\hline $11 / 03 / 2007$ & $15: 50$ & 37.9 & 37.5 & 25 & 7 & 63 & 82 & 100 & 110 & 72 & 88 \\
\hline $11 / 03 / 2007$ & $16: 00$ & 37.9 & 37.5 & 25 & 13 & 63 & 80 & 100 & 110 & 72 & 88 \\
\hline $11 / 03 / 2007$ & $16: 10$ & 37.8 & 37.4 & 25 & 14 & 69 & 79 & 100 & 120 & 74 & 94 \\
\hline
\end{tabular}

Figure 1. 'Screen-shot' of a section of a patient spreadsheet. Data from the bedside data acquisition system is stored to the spreadsheet at intervals of 10 minutes. The figure shows two types of missing data events; at ( $a$ ) data cell is 'empty'; at (b) data is missing due to a 'jump' in the data time-stamp. TMP1, TMP2 denotes temperature parameters; brain temperature $\left({ }^{\circ} \mathrm{C}\right)$ and rectal temperature $\left({ }^{\circ} \mathrm{C}\right)$, respectively. ICP, intra-cranial pressure $(\mathrm{mmHg})$.

acceptable representative (LAR) for the patient gave their informed consent for their relative to participate in the study. Patients of 16 years and above admitted to ICU with severe TBI occurring within 24-hours of injury were included in the study. Patients referred to ICU more than 24 hours after injury were excluded from this acute brain trauma study.

Patients were admitted either as direct referrals from the Emergency department (ED) or as tertiary referrals from EDs of other hospitals within the greater Manchester region. All patients were sedated, intubated and mechanically ventilated and all had an intra- or extra-axial lesion on CT, with or without systemic trauma. Further management was in accordance with local neuro-intensive care guidelines.

Neuro-monitoring at this Centre requires that patients receive dual-modality intra-parenchymal brain monitoring, i.e. intra-cranial pressure (ICP) and temperature $\left(T_{\mathrm{br}}\right)$. The intra-parenchymal sensor (Raumedic ${ }^{\mathrm{TM}}$ Germany) is inserted in to brain tissue via a twist drill burr hole through the skull and dura. The sensor tip is positioned $2-3 \mathrm{~cm}$ into deep white matter, typically in the right (noneloquent) frontal lobe. A small, plastic skull bolt secures the sensor in position. In addition to the neurological parameters monitored for the purpose of this study, patient monitoring included vital signs, core (rectal) temperature, central venous pressure (CVP), oxygen saturation together $\left(\mathrm{PsO}_{2}\right)$ and end tidal $\mathrm{CO}_{2}$. All measurements were displayed via a bedside monitoring system (Marquette, Electronics, Milwaukee, WI). Measurements were displayed in 'real time' on the bedside monitor and also readings were shown at a bedside personal computer and
Table I. Missing data events by category.

\begin{tabular}{lcc}
\hline $\begin{array}{l}\text { Missing data } \\
\text { category }\end{array}$ & $\begin{array}{c}T_{\text {br }} \text { missing } \\
\text { data events, } n(\%)\end{array}$ & $\begin{array}{c}\text { ICP, missing } \\
\text { data events, } n(\%)\end{array}$ \\
\hline MCAR & $364(63)$ & $350(68)$ \\
MAR & $168(29)$ & $158(31)$ \\
MNAR & $46(8)$ & $5(1)$ \\
Total & $578(100)$ & $513(100)$ \\
\hline
\end{tabular}

$\mathrm{T}_{\mathrm{br}}$, brain temperature; ICP, intra-cerebral pressure.

updated at intervals of 10 minutes. At discharge from the ICU, each patient's dataset was exported as an $\mathrm{Excel}^{\mathrm{TM}}$ spreadsheet file (.xls, Microsoft Corporation) for checking and analysis. The completeness of each patients' spreadsheet, specifically for datastreams for $T_{\mathrm{br}}$ and ICP were inspected manually for (a) visible gaps in the 10 minute recording interval, (b) a 'jump' in the spreadsheet time-stamp, (c) unphysiological values and (d) other data 'aberrations' (Figure 1).

After scrutinizing the $T_{\mathrm{br}}$ and ICP data streams of the patient for all events whereby gaps in electronically-derived measurements occurred, the patient's observation charts (mainly recorded by nurses) and medical notes were retrieved to find a possible cause for missing data. The main causes were then classified under the three headings for missing data (Table I), data missing completely at random (MCAR), missing at random (MAR) and missing not at random (MNAR). Each gap in the spreadsheet was considered as one 'missing event', regardless of duration, i.e. the starting time of last complete measurement to the time of the next available measurement. Thus, each 'missing' event is given a 
missing event category only and may vary in terms of duration.

To validate the visual inspection of missing events, three pieces of MATLAB code were written in order to detect missing data and missing data rate. From previous analyses, missing data is typically due to a visible 'gap' in the data stream (type 1 missing data) as well as the number of occasions that data was missing due to a 'jump' in the data spreadsheet timestamp (type 2 missing data). Thus, two different pieces of code were used to detect each type of missing data. A further piece of code was written to calculate the missing data rate: (Number of Type I missing + Number of Type II missing)/Total number of data rows.

\section{Results}

Sixty-two patients, 47 male, 15 female aged 16-75 (median 33) years were included in the study. In over $80 \%$ of patients, brain trauma was due to road traffic accidents (RTA, $n=23(42 \%)$ and falls, $n=22(41 \%))$. In 24 patients the primary brain lesion was subdural haematoma (39\%), followed by subarachnoid haemorrhage $(n=9,14 \%)$, extradural haemorrhage $(n=8,13 \%)$, diffuse axonal injury $(n=7,7 \%)$ and contusions $(n=14,27 \%)$.

In the data sets of the 62 patients with severe TBI, 578 and 513 missing data 'events' were noted in the computer export file for the parameters $T_{\mathrm{br}}$ and ICP, respectively (Table I). The overall percentage of missing data in each of the individual patient's (.xls) spreadsheet ranged from $0.04-72 \%$ (median $25 \%$ ).

The most common category of missing intracerebral data was MCAR (Table I). For $T_{\text {br }} 364$ (63\%) missing data events for the 62 patients were MCAR; 320 (89\%) were due to 'jumps' in the computer export file data time-stamp (Figure 2). For ICP, 350 (68\%) missing data events were MCAR

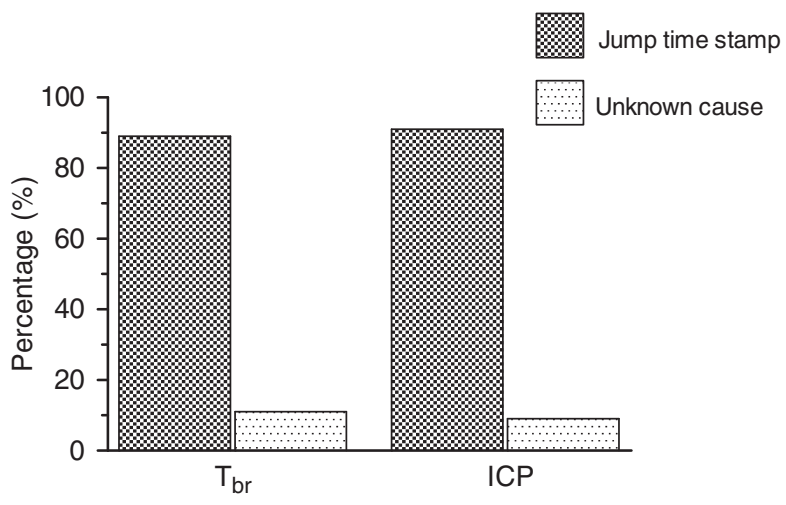

Figure 2. MCAR events for $T_{\mathrm{br}}$ and ICP data due to: a jump in the time-stamp (see text and Figure 1 for explanation) and due to untraceable (and therefore unknown) reasons.
(Table I); 317 events (91\%) due to a jump in the export file data time-stamp (Figure 2).

Further inspection of missing data due to a jump in the export file time-stamp was undertaken for each computer at the 16 ICU bed-spaces (Figure 3). A 'jump' in the database time-stamp from one complete data cell to the next ranged from several minutes of missing data to hours, range 0.17-95.83 hours. Specific 'bed-areas' (5, 6, 7 and 10) were 2-3times more prone to missing data events (Figure 3 ). Unexpected, 'additional' data time-stamps (i.e. more frequently than at 10 minute intervals) were also noted in 25 instances (Figure 3).

In the category of data MAR, the cause for missing data was most commonly attributed to deliberate or accidental disconnection of monitoring equipment during 'critical' medical or nursing care, e.g. during transfer for investigative procedures (CT scan) or surgery (Table II). Within the MAR category there

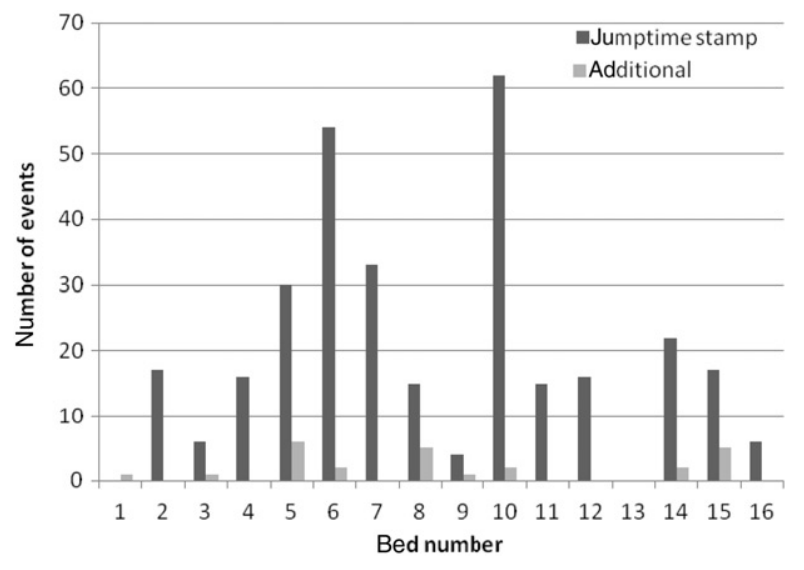

Figure 3. The number of missing data events in the category MAR obtained from data streams at each ICU bed computer with the greatest number of missing events for bed spaces 6 and 10 . Additional data also observed in the data streams in 10 of 16 datasets (beds $1,3,5,6,8,9,10,14,15,16$ ).

Table II. Sources of missing data in the category MAR for brain temperature and intracranial pressure, values represent number of missing data events.

\begin{tabular}{lrr}
\hline & $\mathrm{T}_{\mathrm{br}}$ & ICP \\
\hline Transfer to Radiology for CT scan & 69 & 69 \\
Data recording stopped (bed transfer) & & 28 \\
Transfer to operating room & 26 & 26 \\
$\begin{array}{l}\text { Delay in data storage following insertion } \\
\quad \text { of monitoring device }\end{array}$ & 33 & 29 \\
$\quad$ Interruption in monitoring due to & 32 & 6 \\
$\quad$ patient-specific event & 9 & 0 \\
Seizure & 169 & 158 \\
\hline Total & & \\
\hline
\end{tabular}

$\mathrm{T}_{\mathrm{br}}$, brain temperature; ICP, intra-cerebral pressure. 
were 62 occasions where there was a 'data lag', i.e. the data input entry time began before the sensor was connected to the monitor. Missing data 'events' in the category MNAR occurred for 46 events ( $8 \%$ ) for $T_{\mathrm{br}}$ and for five events (1\%) for ICP (Table III). In this category, data in the export file was removed by the researcher on the basis of being 'unphysiological' or biologically impossible.

\section{Discussion}

The challenge for researchers, as has been observed in this series of brain tissue monitoring data sets, is that where visual inspection might initially appear to be all that is required to indentify 'empty data cells', the opposite may be true; greater scrutiny of the data streams are required to determine the completeness of each data set. In this cohort of patients with severe TBI, an average of $25 \%$ of data was missing from each of the 62 patients' spreadsheets. On initial checking of each spreadsheet, the majority of columns and rows appeared 'complete', with few empty cells. However, rigorous inspection of the data to check for consistency in the electronic data record revealed the frequency with which data was missing. By reference to the literature on sources of 'missingness' [8], the authors were able to categorize these missing data events to the categories MCAR, MAR and MNAR. By far the largest category was MCAR, accounting for $60 \%$ of missing data and composed predominately of what has been defined as data time-stamp 'jump'. Such events were shown to be unpredictable in occurrence and in duration and, as a consequence, the accompanying brain tissue monitoring readings were lost from the dataset. Knowledge that export file spreadsheets may be incomplete has allowed one to highlight the nature and source of the missing data and now guides the method for dealing with incomplete data sets before analysis is undertaken. By running a simple computer program to the export files, the authors were able to identify and thus 'validate' the visually inspected data streams with ease, but identifying sources of error are certainly more time-consuming.

When data are MCAR, the main consequence of this type of 'missingness' is loss of statistical power. For data MAR, if the causes of the missing values are not taken into account, the statistical inferences and predictions can be biased or even totally wrong. Here different methods have been studied in the literatures by methodologists. For example imputation has been shown to give more accurate outcome results than if a 'zero' value is used. However, for datasets that are MNAR, using the above methods could introduce misleading results [7, 12]. More advanced statistical approaches need to be developed to account for this complex missing data mechanism.

Within the timeframe of this study it was possible to identify specific reasons for the occurrences of spontaneous jumps in the electronic time-stamp that could be attributed to specific TBI patient characteristics. The use of imputation for events in the MCAR category is safely indicated [7]. However, that said, a limitation of this study is that we considered a 'jump time-stamp' as a 'single' event even though the duration for missing data was variable (data not shown). The effect of data imputation on the analysis of temporal profiles of brain temperature and intracranial pressure in patients with severe TBI, particularly with respect to standard clinical correlates has not, as far as we are able to ascertain, been investigated before.

In this context, one now needs to acknowledge that an important source of missing data occurs at a time when knowledge of changes in brain tissue homeostasis may be of most importance, i.e. during

Table III. Categories of missing data with explanation for missing reading events after cross-reference to text documented in nursing observation sheets and medical case notes.

\begin{tabular}{ll} 
Category & \multicolumn{1}{c}{ Examples of missing data events } \\
\hline$M C A R$ & $\begin{array}{l}\text { Jump time-stamp: i.e. recordings missing for }>10 \text { minutes, with no gaps seen on spreadsheet (see } \\
\text { Figure 1) } \\
\text { Unknown reason for missing data }\end{array}$ \\
$\begin{array}{l}\text { MAR } \\
\text { Deliberate disconnection } \\
\text { from monitor }\end{array}$ & $\begin{array}{l}\text { Patient transferred from ward for radiology investigations, e.g. CT scan } \\
\text { Patient transferred to operating department for surgery }\end{array}$ \\
$\begin{array}{l}\text { Accidental disconnection } \\
\text { Deliberate disconnection of sensors or cables from monitoring system stopped }\end{array}$ & $\begin{array}{l}\text { Data missing during a specific clinical intervention or patient care but explanation unclear } \\
\text { Time lag from monitor switch-on to connection of patient monitoring devices (typically at time of } \\
M N A R\end{array}$ \\
admission to ICU) & Values recorded on spreadsheet but subsequently removed on the basis of 'unphysiological' readings
\end{tabular}


'critical clinical events'. Cross-reference to the case notes and daily observation sheets revealed that 30\% of missing data occurred at or around the time of patient transfer for a CT scan, surgery and, more importantly from the clinical perspective, during documented patient deterioration. Since such sources of missing data are specific to individual patients, they are categorized as data missing at random (MAR). Caution is needed, however, when dealing with this type of data, as there is a potential for bias. In reviews on handling of missing data, dropping cases where data is MAR could alter the robustness of the interpretation of data, especially in cases where data values are used in prognosis [13].

The percentage of MNAR data constituted a relatively low proportion of missing cells. When data is MNAR, the probability of data missing depends on the unobserved variable itself and therefore is not ignorable. In this case, the values obtained were deemed unphysiological, e.g. $T_{\mathrm{br}}$ of $24^{\circ} \mathrm{C}$, when all other variables were within the physiological range. However, even though those values could not be included in this research and were hence considered as 'missing', the origin of missingness could well be attributed to an unknown specific patient characteristic at the time. Data that is MNAR is problematic as there is potential to introduce bias. Models specific to the data involved need to be designed to handle MNA [13].

Digital export files saved in spreadsheet format are a convenient means to gather patient data. Loss of data, however, can be a significant problem which, without robust scrutiny of the spreadsheet, could bias the interpretation of results. In this study the missing data rate was variable at $9-43 \%$ (median $25 \%$ ). Utilizing existing hospital data acquisition systems during clinical research studies requires attention to the potential sources of analysis bias due to the finding that, as in this case, as much as $40 \%$ of the data could be missing from the export file. This study has shown that gaps in the dataset are not the only 'identifiers' of an incomplete data record; electronic time-stamp jumps will often go un-noticed and, importantly, mask missing data for long periods of time. This is an observation of relevance to all areas of clinical research data collection, alerting clinicians to the need to scrutinize data for potential sources of 'missingness'. A limitation to this study might be levelled at the accuracy of the clinical charts to convey the reasons for missing data but this is a valid point and a justification for future investigation in a larger patient series where, in the climate of the 'paperless' ICU, more emphasis will be on the accuracy and reliability of periodic electronic data.

\section{Conclusions}

Missing data is common in electronic periodic data acquired from bedside monitoring systems with $60 \%$ of missing intracerebral (brain temperature and intra-cranial pressure) data missing due to electronic data acquisition 'faults'. On average, $25 \%$ of data was lost from the data set and, most importantly for evidence-based practice and reliable inferences during monitoring, the 'lost' data occurred during clinical events which are recognized as 'high risk'. Disconnection of cables from the monitoring system, whether accidental or deliberate, may represent a significant threat to the validity of acquired clinical data. Of importance in this population of patients with severe brain injury is the concern that any sudden changes in ICP or brain temperature (the parameters which forewarn of impending intracranial catastrophes) will be missing at the most critical times of the patients critical care monitoring.

\section{Acknowledgements}

We thank Mr Timothy Rainey for his technical assistance during the course of this work and Mr Martin Ogden, Salford Royal Foundation Trust for assistance in preparing the database.

Declaration of interest: The authors report no conflicts of interest. The authors alone are responsible for the content and writing of the paper.

\section{References}

1. Tagliaferri FC, Compagnone C, Korsic M, Servaei F, Kraus J. A systematic review of brain epidemiology in Europe. Acta Neurochirurgica (Wien) 2006;148:225-268.

2. Turner-Stokes L. Evidence for the effectiveness of multidisciplinary rehabilitation following acquired brain injury: A synthesis of two systematic approaches. Journal of Rehabilitation Medicine 2008;40:691-701.

3. Childs C, Wieloch T, Lecky F, Machin G, Harris B, Stocchetti N. Report of a consensus meeting on human brain temperature after severe traumatic brain injury: Its measurement and management during pyrexia. Frontiers in Neurotrauma 2010;1:146.

4. Maas AIR, Stocchetti N, Bullock R. Moderate and severe traumatic brain injury in adults. The Lancet Neurology 2008;8:728-741.

5. Childs C, Machin G. Reliability issues in human brain temperature measurement. Critical Care 2009;13:106.

6. Childs C, Vail A, Leach P, Rainey T, Protheroe R, King AT. Brain temperature and outcome after severe traumatic brain injury. Neurocritical Care 2006;05:10-14.

7. Gorelick MH. Bias arising from missing data in predictive models. Journal of Clinical Epidemiology 2006;59:1115-1123.

8. Curran D, Bacchi M, Schmitz S, Molenberghs G, Sylvester R. Identifying the types of missingness in quality of life data from clinical trials. Statistics in Medicine 1998;17:739-756. 
9. Rubin DB. Inference and missing data. Biometrika 1976;63: 581-592.

10. Heitjan DF, Basu S. Distinguishing missing at random and missing completely at random. The American Statistician 1996;50:207-213.

11. Sterne JC, White IR, Carlin JB, Spratt M, Royston P, Kenward MG, Wood AM, Carpenter JR. Multiple imputation for missing data in epidemiological and clinical research. British Medical Journal 2009;338: b2393.
12. Norris CM, Ghali WA, Knudtson ML, Naylor CD, Saunders LD. Dealing with missing data in observational health care outcome analyses. Journal of Clinical Epidemiology 2000;53:377-383.

13. Hamilton BH, Ko CY, Richards K, Hall BL. Missing data in the American College of Surgeons National Surgical Quality Improvement Program are not missing at random: Implications and potential impact on quality assessments. Journal of the American College of Surgeons 2010;210: 125-139. 